\title{
Trends of serum phospholipid fatty acids over time in rural Uganda: evidence of nutritional transition?
}

\author{
Véronique Chajès ${ }^{1 *}$, Lorna J. Gibson ${ }^{2}$, Carine Biessy ${ }^{1}$, Nadia Slimani ${ }^{1}$, Gershim Asiki ${ }^{3}$, Laure Dossus ${ }^{1}$, \\ Christopher P. Wild ${ }^{4}$ and Robert Newton ${ }^{3,5} \dagger$ \\ ${ }^{1}$ Section of Nutrition and Metabolism, International Agency for Research on Cancer, 69382 Lyon Cedex 08, France \\ ${ }^{2}$ Department of Social and Environmental Health Research, London School of Hygiene and Tropical Medicine, London \\ WC1E $7 H T$, UK \\ ${ }^{3}$ Epidemiology and Prevention Programme, MRC/UBRI, Entebbe, Uganda \\ ${ }^{4}$ International Agency for Research on Cancer, 69372 Lyon Cedex 08, France \\ ${ }^{5}$ Department of Health Sciences, University of York, York YO1O 5DD, UK
}

(Submitted 22 June 2018 - Final revision received 28 September 2018 - Accepted 9 October 2018)

\section{Abstract}

Non-communicable diseases are projected to become the most common causes of death in Africa by 2030. The impact on health of epidemiological and nutritional transitions in sub-Saharan Africa remains unclear. To assess the trends of dietary fatty acids over time in Uganda, we examined fatty acids in serum collected from individuals in rural south-west Uganda, at three time points over two decades. Independent cross-sectional samples of 915 adults and children were selected from the general population cohort in 1990 ( $n$ 281), 2000 ( $n$ 283) and 2008 ( $n$ 351). Serum phospholipid fatty acids were measured by GC. Multivariate regression analyses were performed to compare the geometric means of fatty acids by time period. Serum fatty acid profiling showed high proportions of SFA, cis-MUFA and industrial transfatty acids (iTFA), likely to be biomarkers of high consumption of palm oil and hydrogenated fats. In contrast, proportions of $n$ - 6 and $n$ - 3 PUFA from vegetable oils and fish were low. From 1990 to 2008, serum phospholipids showed increases in absolute amounts of SFA (17.3\% increase in adults and $26.4 \%$ in children), MUFA ( $16.7 \%$ increase in adults and $16 \cdot 8 \%$ in children) and $n-6: n-3$ PUFA (40.1\% increase in adults and $39.8 \%$ in children). The amount of elaidic acid, iTFA from hydrogenated fats, increased in children (60.1\% increase). In this rural Ugandan population, we show evidence of unfavourable trends over time of dietary fatty acids.

Key words: Nutrition transition: Fatty acids: Biomarkers: Epidemiology: East Africa

Non-communicable diseases (NCD) are projected to become the most common causes of death in Africa by $2030^{(1)}$. Despite this fact, risk factors - including nutritional factors - have not been fully studied in African populations undergoing epidemiological and nutritional transitions, with concomitant changes in lifestyle and diet. In high-income countries, diets began to shift in the 1970s, from a traditional pattern of high carbohydrate and fibre and low fat and sugar, toward a more globalised 'Western' pattern characterised by increased consumption of processed foods, greater use of edible oils and sugar-sweetened beverages ${ }^{(2)}$. Similar changes started to emerge in the 1990s in low- to middle-income countries ${ }^{(2-4)}$. The nutritional transition has led to an increased consumption of products that can be harmful to health at high levels, such as sugar, Na and fat, particularly SFA and industrial trans-fatty acids (iTFA). In turn, this has had an impact on patterns of cancers, from one dominated by infection-related cancers to one characterised by cancers related to changing reproductive patterns, poor diet, excess body fatness and reduced physical activity ${ }^{(5)}$.

Countries in sub-Saharan Africa are clearly undergoing a nutrition transition ${ }^{(3)}$. More than half of them are still in the early stage, as illustrated by the finding that fat, protein and carbohydrate intake are still within those recommended, while a few have already reached a situation where changes in dietary patterns are affecting health outcomes in the population ${ }^{(3,6)}$. Data from the FAO indicated a steady increase in the energy supply in most sub-Saharan countries over a period of three decades, protein and fat intake displaying a similar trend ${ }^{(7)}$. An increase in fat consumption has been shown to be highly associated with global dietary changes ${ }^{(3)}$. Thus, these data suggest that measurement of fatty acid intakes might serve as an indicator of nutrition transition in general.

Abbreviations: GPC, general population cohort; iTFA, industrial trans-fatty acid; NCD, non-communicable disease; TFA, trans-fatty acid.

* Corresponding author: V. Chajès, fax +33472738361, email chajesv@iarc.fr

$\dagger$ R. N. is a Senior Visiting Scientist at the International Agency for Research on Cancer. 
Measurement of dietary fatty acids in nutritional epidemiology studies has been hampered by substantial measurement error when using dietary questionnaires. Plasma or serum phospholipid fatty acid level measurements offer specific biomarkers of medium-term (weeks to months) dietary intake of bioavailable amounts of these nutrients, particularly for fatty acids that are not endogenously synthesised, such as iTFA and $n-6$ and $n-3$ PUFA $^{(8,9)}$. Thus, the use of specific biomarkers of fatty acids is a more rigorous approach to provide a better understanding of changes in dietary fatty acids in low- to middle-income countries.

The general population cohort (GPC) set-up in rural southwestern Uganda provided a unique framework to examine the biomarkers of dietary fatty acids. We measured serum phospholipid fatty acid levels at three time periods, in 1990-1991, 1999-2000 to 2007-2008, and monitored changes over this period in a representative sample within the GPC.

\section{Methods \\ Population}

The GPC was set up as a population-based open cohort study established in 1989 to examine trends in HIV prevalence and incidence in rural south-western Uganda. Since 2010, the scientific research questions have incorporated the epidemiology and genetics of both communicable and NCD. The study area is located in Kyamulibwa sub-county of Kalungu district. The initial study population of about 10000 residents comprised a cluster of fifteen neighbouring villages. From 1999, ten more adjacent villages with comparable characteristics were added to the cohort, thereby doubling the population ${ }^{(10)}$.

The study population was recruited through annual house-tohouse rounds of census through which participants for the medical survey are selected. All residents aged 13 years and above were included in all the medical survey rounds 1-22 (1989-2011). Children $<13$ years were also recruited every third round. Data on health and lifestyle are collected using a standard individual questionnaire, and blood samples were obtained and biophysical measurements taken. Blood samples were transported to MRC/UVRI Laboratories in Entebbe and stored at $-80^{\circ} \mathrm{C}$. Overall, more that $95 \%$ of households approached for census participated.

\section{Ethics statement}

Study participants provided written informed consent to participate in the study. Ethical approval for this study was granted by London School of Hygiene and Tropical Medicine and the Uganda Virus Research Institute Research and Ethics Committee, and the study was approved by the Uganda National Council for Science and Technology. It was also approved by the Ethics Committee of the International Agency for Research on Cancer (IARC).

\section{Selection criteria}

Census rounds 3,11 and 19 were chosen because of the inclusion of children. A total of three independent random samples of 281 individuals (149 adults and 132 children), 283 individuals (166 adults and 117 children) and 351 individuals (238 adults and 113 children) were selected on the basis of availability of serum sample and stratified to provide a 1:1 sex ratio. Serum samples were shipped to the IARC in Lyon, France.

\section{Laboratory analysis}

All measurements of fatty acids were performed at the IARC. Serum samples were profiled for phospholipid fatty acid composition by batches of twenty samples, including samples from eighteen subjects at three time periods (six men, six women and six children) in random order, and two independent serum samples as quality controls. The laboratory staff was blinded to the status of the sample (subject, sex, time period or quality control). As previously described, total lipids were extracted from serum samples, phospholipids were purified by adsorption chromatography, and fatty acid methyl esters separated through $\mathrm{GC}^{(11)}$. The relative concentration of each fatty acid, expressed as percentage of total fatty acids, was quantified by integrating the area under the peak and dividing the result by the total area. Fatty acids were also expressed as absolute concentrations in serum $(\mu \mathrm{mol} / \mathrm{l})$.

Overall (intra-batch and inter-batch) CV for fatty acids, which were calculated using two serum samples as quality controls added to each batch, ranged from $0.29 \%$ for large peaks such as palmitic acid, to $7 \cdot 74 \%$ for the smallest peaks such as 18:3trans. Specifically for trans-fatty acids (TFA), the overall CV were 0.112 for $16: 1 n$-9trans, 0.137 for $18: 1 n$-9trans, 0.376 for $18: 2$ trans, cis, 0.198 for $18: 2$ trans,trans, 7.74 for $18: 3 n$ 3 trans,cis,cis, 0.282 for $18: 1 n$-7trans, 1.017 for CLA 10trans,12cis and 0.097 for CLA 9trans,11cis.

\section{Statistical analysis and data treatment}

Multivariate regression analyses were performed to compare the geometric mean levels (mean 95\% confidence intervals) of fatty acids by time period. We calculated the percentage and absolute amounts of the following groups: SFA, cis-MUFA, ruminant TFA, iTFA, cis- $n$-6 PUFA, long-chain $n$-6 PUFA, $n$-3 PUFA, long-chain $n-3$ PUFA and the ratio of $n-6: n-3$ PUFA. Analyses were conducted separately in children, men and women and in men and women combined. Tests for trend were computed using the time period-specific geometric means of each fatty acid. The models were adjusted for age at baseline for men and for women separately. For children and for adults combined, the models were adjusted for age at baseline and for sex. All analyses were performed with STATA version 13 software.

\section{Results}

\section{Subject characteristics}

Characteristics of the study population are described above and in a previous report ${ }^{(10)}$. Briefly, the cohort includes all residents ( $52 \%$ aged $\geq 13$ years, men and women in equal proportions) within one-half of a rural sub-county, residing in scattered houses. Characteristics of the study population showed a 
relatively young population, with about $90 \%$ of the population $<50$ years old, predominantly farmers of three major ethnic groups. Only $13 \%$ of the population attained education beyond primary level.

\section{Serum phospholipid fatty acid profile}

The separation of serum phospholipid fatty acids, particularly TFA isomers, is shown in Fig. 1. The serum phospholipid fatty acid profile in the adult study population at baseline (19901991) is provided in Table 1. Due to a lack of appropriate reference values, we used serum phospholipid fatty acid profiles reported at the same time in other cross-sectional studies of healthy adults to determine whether our population had been exposed to low or high fatty acid levels. Serum phospholipid fatty acid proportions reported in the European Prospective Investigation into Cancer and Nutrition (EPIC) study ${ }^{(8,9)}$, the Japan Public Health centre-based prospective study on cancer and CVD study ${ }^{(12)}$ and the New Zealand National Nutrition Survey ${ }^{(13)}$ are provided for comparison. In all studies, fatty acids are expressed in percentage of total fatty acids. Because the proportions of fatty acids are similar in adult men and women in the Ugandan population study (data not shown), combined data are provided for men and women.

In 1990-1991, the fatty acid profile in Ugandan adults is characterised by a high proportion of SFA ( $44.53 \%$ of total fatty acids), mainly palmitic acid (27.56\%). More striking is the very high proportion of cis-MUFA (26.30\%), mainly oleic acid (20.50\%). In contrast, the proportions of $n-6$ PUFA (21.66\%), mainly linoleic acid (12.59\%) and $n-3$ PUFA (3.22\%) are low. The ratio of $n-6 . n-3$ PUFA is 6.73 . The proportion of TFA from industrial processing $(1.72 \%)$ is relatively high, mainly as the consequence of a high proportion of palmitelaidic acid, sixteen-carbon TFA (Fig. 1). In contrast, the proportion of elaidic acid, the main TFA from industrial processing, is relatively low, as is the proportion of TFA from natural sources mainly represented by vaccenic acid.

\section{Trends in serum fatty acids over time}

Trends in serum phospholipid fatty acids, expressed in absolute amounts and in proportions, from 1990 to 2008 are shown in Table 2 for adults and children.

Notably, there were statistically significant increases in absolute amounts of SFA (17.3\% increase in adults and $26.4 \%$ in children) (mainly palmitic acid), of cis-MUFA ( $16.7 \%$ increase in adults and $16.8 \%$ in children) (mainly oleic acid) and of total $n$-6 PUFA (20.3\% increase in adults and $33.8 \%$ in children), as a consequence of the increasing levels of linoleic acid and longchain $n-6$ PUFA. These trends were present in children and adults but were stronger in children; an unexplained peak level of SFA and MUFA was seen in adults in the 1999-2000 collection. There was an increasing ratio of $n-6: n-3$ PUFA, rising to (a)

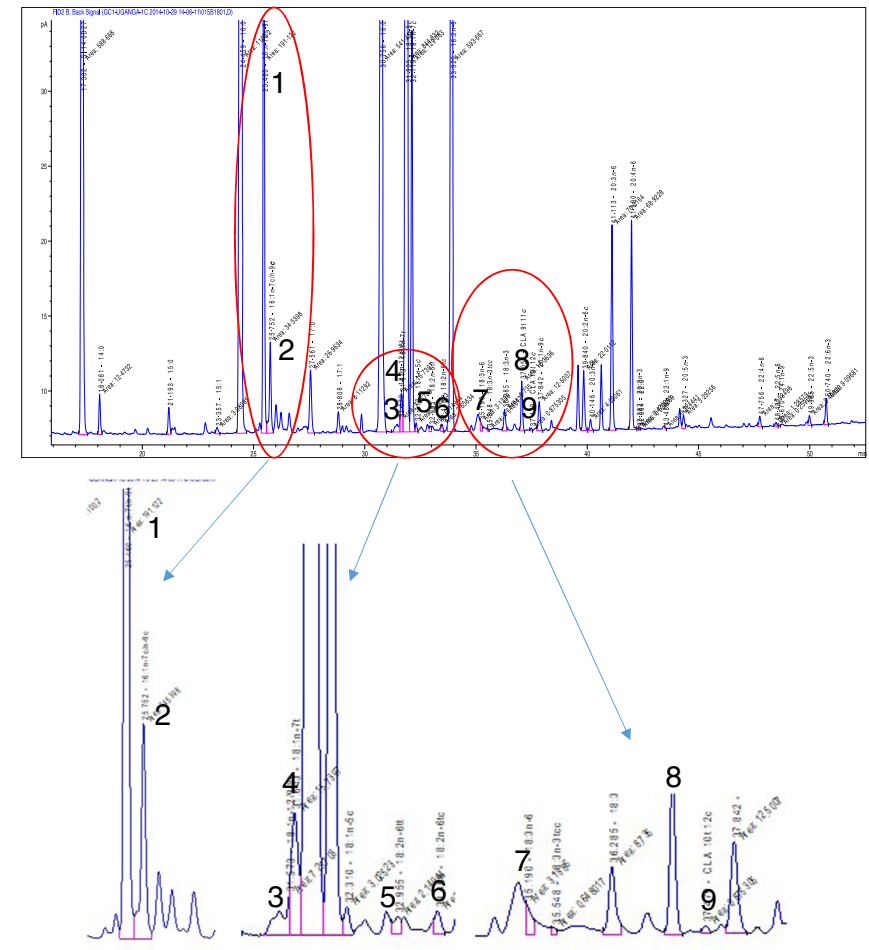

(b)
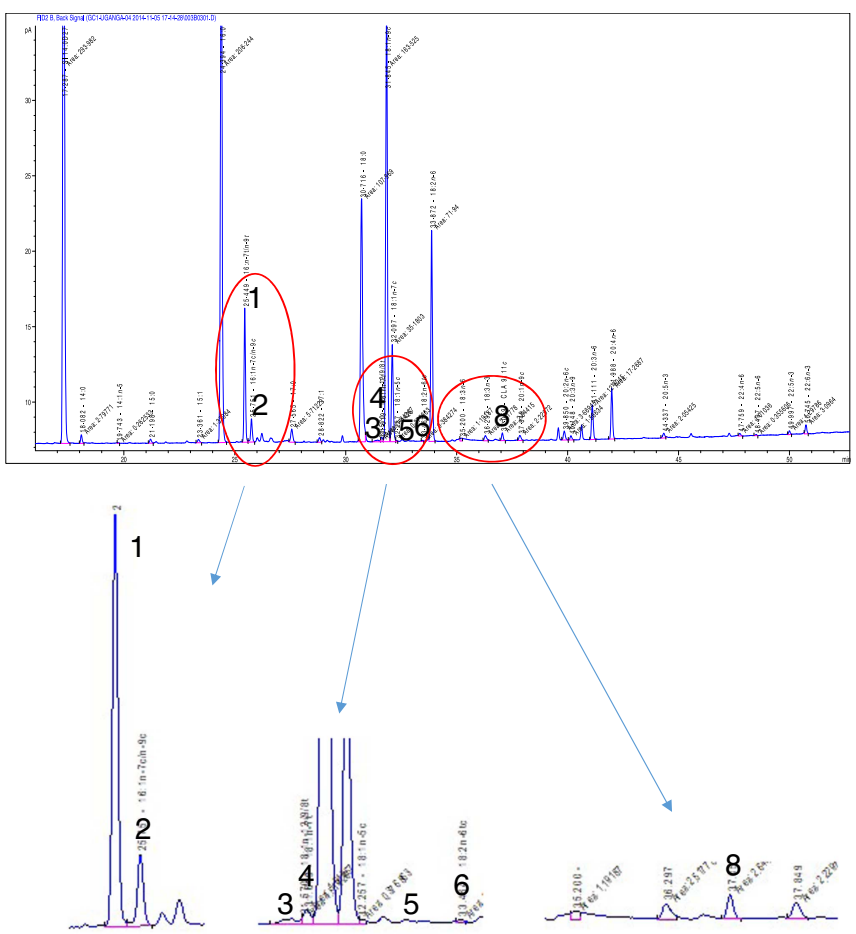

Fig. 1. Separation of serum phospholipid fatty acids through GC. (a) The separation of fatty acids in a sample from the population study with a high proportion of transfatty acid isomers, while (b) shows a sample with a low proportion of trans-fatty acid isomers. 1, Palmitelaidic acid (16:1n-9trans); 2, palmitoleic acid (16:1n-9cis); 3 , elaidic acid (18:1n-9trans); 4, vaccenic acid (18:1n-7trans); 5 and 6 , linolelaidic acid isomers (18:2n-6trans, trans, 18:2n-6trans,cis); 7, linolenelaidic acid (18:3n-3trans,cis,cis); 8, conjugated linoleic acid (CLA) 9trans,11 cis; 9, CLA 10trans,12cis. Industrial trans-fatty acids (iTFA) included peaks $1,3,5,6$ and 7, while ruminant trans-fatty acids included peaks 4,8 and 9 . 
Table 1. Serum phospholipid fatty acid ranges in the general population cohort (GPC) in Uganda, the European Prospective Investigation into Cancer and Nutrition study, the Japan Public Health centre-based prospective study on cancer and CVD study and a New Zealand National Nutrition Survey (Mean values, 95\% confidence intervals and ranges)

\begin{tabular}{|c|c|c|c|c|c|c|c|c|}
\hline \multirow[b]{2}{*}{ Fatty acids (\% total fatty acids) } & \multicolumn{2}{|c|}{$\begin{array}{l}\text { Adults, GPC Uganda } \\
(1990 ; n \text { 149) }\end{array}$} & \multicolumn{2}{|c|}{$\begin{array}{c}\text { Adults, Europe }{ }^{(8)} \\
(1992-1998 ; n \text { 3262) }\end{array}$} & \multicolumn{2}{|c|}{$\begin{array}{l}\text { Adults, Japan }{ }^{(12)} \\
(1994-1995 ; n \text { 87) }\end{array}$} & \multicolumn{2}{|c|}{$\begin{array}{l}\text { Adults, New Zealand }{ }^{(13)} \\
(1993-1998 ; n \text { 2416) }\end{array}$} \\
\hline & Mean & $95 \% \mathrm{Cl}$ & Mean & $95 \% \mathrm{Cl}$ & Mean & Range & Mean & Range \\
\hline Total SFA & 44.53 & $44.12,44.93$ & $40 \cdot 46$ & $40.38,40.53$ & $47 \cdot 60$ & $42 \cdot 20-58 \cdot 90$ & $50 \cdot 25$ & $43 \cdot 19-61 \cdot 22$ \\
\hline Palmitic acid $(16: 0)$ & $27 \cdot 56$ & $27 \cdot 11,28.01$ & $25 \cdot 41$ & $25 \cdot 33,25 \cdot 49$ & $28 \cdot 70$ & $23 \cdot 20-36 \cdot 90$ & 31.73 & $28.41-37.56$ \\
\hline Stearic acid $(18: 0)$ & 15.55 & $15 \cdot 19,15 \cdot 91$ & 13.99 & $13.94,14.05$ & $15 \cdot 10$ & $11 \cdot 70-20 \cdot 80$ & 14.34 & $11.95-17.79$ \\
\hline Total cis-MUFA & $26 \cdot 30$ & $25 \cdot 56,27 \cdot 07$ & $12 \cdot 80$ & $12 \cdot 72,12 \cdot 88$ & $13 \cdot 60$ & $10 \cdot 30-18 \cdot 40$ & $12 \cdot 56$ & $9.04-16.85$ \\
\hline Palmitoleic acid (16:1n-9cis) & 1.32 & $1.24,1.40$ & 0.60 & $0.59,0.60$ & & NA & 0.83 & $0.38-1.43)$ \\
\hline Oleic acid $(18: 1 n-9 \mathrm{cis})$ & $20 \cdot 50$ & $19 \cdot 85,21 \cdot 16$ & $10 \cdot 09$ & $10 \cdot 02,10 \cdot 17$ & 8.80 & $6 \cdot 30-12 \cdot 60$ & $10 \cdot 00$ & $7.57-13.00$ \\
\hline Total cis- $n-6$ PUFA & 21.66 & $20 \cdot 93,22 \cdot 41$ & $37 \cdot 38$ & $37 \cdot 25,37.51$ & $26 \cdot 50$ & $15 \cdot 30-33 \cdot 20$ & $26 \cdot 26$ & $18 \cdot 73-38.00$ \\
\hline Linoleic acid (18:2n-6cis,cis) & 12.59 & $12 \cdot 03,13 \cdot 19$ & 21.83 & $21 \cdot 63,22 \cdot 02$ & $17 \cdot 30$ & $9 \cdot 30-24 \cdot 30$ & $19 \cdot 01$ & $12.72-24.54$ \\
\hline Long-chain $n$-6 PUFA & $8 \cdot 76$ & $8 \cdot 41,9 \cdot 12$ & $15 \cdot 17$ & $15 \cdot 07,15 \cdot 26$ & & NA & $7 \cdot 25$ & $6.01-13.46$ \\
\hline Total cis-n-3 PUFA & 3.22 & $2.99,3.47$ & 6.90 & $6.83,6.97$ & $12 \cdot 40$ & $7 \cdot 00-20 \cdot 20$ & 4.74 & $2.31-7.59$ \\
\hline$a$-Linolenic acid $(18: 3 n-3 \mathrm{cis}, \mathrm{cis}, \mathrm{cis})$ & 0.15 & $0.14,0.17$ & 0.18 & $0.18,0.19$ & 0.20 & $0.10-1 \cdot 20$ & 0.24 & $0.12-0.42$ \\
\hline Long-chain $n-3$ PUFA & $2 \cdot 84$ & $2.63,3.06$ & $6 \cdot 69$ & $6 \cdot 62,6 \cdot 76$ & $12 \cdot 20$ & $5 \cdot 80-20 \cdot 80$ & 4.50 & $2 \cdot 19-7 \cdot 17$ \\
\hline$n-6: n-3$ PUFA & 6.73 & $6 \cdot 21,7 \cdot 29$ & 5.42 & $5 \cdot 36,5.48$ & $2 \cdot 30$ & $0.80-4.40$ & 5.54 & $5 \cdot 01-8 \cdot 11$ \\
\hline Total industrial trans-fatty acids & 1.72 & $1.58,1.86$ & 0.48 & $0.47,0.49$ & & NA & & NA \\
\hline Palmitelaidic acid (16:1n-9trans) & 1.28 & $1.16,1.41$ & 0.28 & $0.27,0.29$ & & NA & & NA \\
\hline Elaidic acid (18:1n-9trans) & 0.18 & $0.16,0.19$ & 0.31 & $0.30,0.31$ & & NA & & NA \\
\hline Total natural trans-fatty acids & 0.22 & $0.19,0.25$ & 0.80 & $0.79,0.82$ & & NA & & NA \\
\hline Vaccenic acid (18:1n-7trans) & 0.08 & $0.06,0.09$ & 0.26 & $0.25,0.27$ & & NA & & NA \\
\hline
\end{tabular}

NA, not available.

9.74:1 and $8 \cdot 43: 1$ in adults (40.1\% increase) and children (39.8\% increase), respectively, in 2007-2008. There was a significant increase (of around $60 \%$ ) in the mean level of elaidic acid, the main eighteen-carbon monoenoic TFA isomer from hydrogenated fats in children, with far less of a rise in adults. In contrast, the levels of palmitelaidic acid showed a decreasing trend in adults ( $9 \%$ decrease) and children ( $8 \%$ decrease). TFA from natural animal sources remained very low over time.

Expression of fatty acids, both in proportions and in absolute amounts, showed similar directions of changes over time; however, changes are generally more pronounced when expressed in absolute amounts (Table 2).

\section{Discussion}

This study provides the first direct evidence of the transition in serum fatty acid profiles in a rural African population over time. The profile is characterised by high proportions of SFA, cisMUFA and iTFA, low proportions of PUFA along with a high ratio of $n-6: n-3$ PUFA. This profile persisted from 1990 to 2008, but with increases in the absolute concentrations of SFA, MUFA, $n-6$ PUFA and the ratio of $n-6: n-3$ PUFA. A specific TFA isomer from industrial processing increased in children. Current profiles show high levels of fatty acids that are known to be deleterious to health.

Compared with data reported for adults in Europe ${ }^{(8)}$, Japan $^{(12)}$ and New Zealand ${ }^{(13)}$, the fatty acid profile in Ugandan adults in 1990-1991 is markedly different. The most prominent difference in Uganda is the far higher proportion of MUFA, mainly oleic acid. The Ugandan population is also characterised by higher proportions of SFA, mainly palmitic acid, than that reported in Europe, close to Japan and lower than New Zealand. The proportion of $n-6$ PUFA in Uganda is the lowest of the four populations compared. Similarly, the proportion of $n-3$ PUFA is much lower in Uganda than in the three other populations, particularly Japan. These differences result in Uganda having the highest ratio of $n-6: n-3$ PUFA. Among the different TFA isomers, the proportion of palmitelaidic acid, sixteencarbon monoenoic $n-9$ TFA from industrial processing, is much higher in Uganda than in Europe, while the proportion of elaidic acid, the main eighteen-carbon monoenoic n-9 TFA from industrial processing, is lower in Uganda than in EPIC. The proportion of TFA isomers from dairy product intake is much lower in Uganda than in Europe.

High proportions of SFA palmitic acid and MUFA oleic acid in the GPC in Uganda are likely to be the consequence of high consumption of palm oil rich in palmitic acid and oleic acid, commonly used for cooking in Africa ${ }^{(14)}$. High proportions of TFA from industrial processing are likely to be the consequence of high dietary intake of palmitelaidic acid, TFA isomer from partially hydrogenated vegetable oils (PHVO) - these are also used for cooking as well as being added to a myriad of processed foods to cheaply improve the shelf life and palatability. Major dietary sources of this specific TFA isomer are deep-fried foods, bakery products, packaged snack foods, margarines ${ }^{(7,15,16)}$ and also heating/frying and reuse of edible fats/ oils $^{(17)}$. Low proportions of natural TFA in the population study are likely to be the consequence of low consumption of dairy foods. Low proportions of n-6 PUFA likely result from low intake of vegetable oils rich in $n-6$ PUFA, as reported in a previous cross-sectional study within the GPC ${ }^{(18)}$. Finally, low proportions of long-chain $n-3$ PUFA may reflect low intake of fish $^{(8)}$.

Trends in the levels of fatty acids from 1990 to 2008 in the population study showed significant increases in SFA (particularly in children), mainly palmitic acid, cis-MUFA, mainly oleic acid, $n$-6 PUFA, along with an increased ratio of $n-6: n-3$ PUFA, 
Table 2. Evolution trends of serum phospholipid fatty acids over time in the general population cohort, Uganda (Mean values and $95 \%$ confidence intervals)

\begin{tabular}{|c|c|c|c|c|c|c|c|c|c|c|c|c|c|}
\hline \multirow[b]{2}{*}{ Fatty acids $(\mu \mathrm{mol} / \mathrm{l})$} & \multirow[b]{2}{*}{ Time period } & \multicolumn{6}{|c|}{ Adults } & \multicolumn{6}{|c|}{ Children } \\
\hline & & Mean $(\mu \mathrm{mol} / \mathrm{l})$ & $95 \% \mathrm{Cl}$ & $P_{\text {trend }}$ & Mean (\%) & $95 \% \mathrm{Cl}$ & $P_{\text {trend }}$ & Mean $(\mu \mathrm{mol} / \mathrm{l})$ & $95 \% \mathrm{Cl}$ & $P_{\text {trend }}$ & Mean (\%) & $95 \% \mathrm{Cl}$ & $P_{\text {trend }}$ \\
\hline \multirow[t]{3}{*}{ Total SFA* } & 1990-1991 & $1040 \cdot 82$ & $944.84,1146.54$ & & 44.53 & $43.41,45 \cdot 67$ & & $1087 \cdot 15$ & $987 \cdot 6,1196 \cdot 75$ & & $45 \cdot 11$ & $44.69,45.53$ & \\
\hline & 1999-2000 & $1370 \cdot 70$ & $1250 \cdot 7,1502 \cdot 3$ & & 44.62 & $43 \cdot 56,45 \cdot 70$ & & 1247.59 & $1126 \cdot 6,1381 \cdot 6$ & & 46.52 & $46.06,46.99$ & \\
\hline & 2007-2008 & 1221.04 & $1131.06,1318.2$ & 0.037 & 44.75 & $43.86,45 \cdot 66$ & 0.752 & 1374.43 & $1238.9,1524.8$ & 0.001 & 46.02 & $45.55,46.49$ & 0.003 \\
\hline \multirow[t]{3}{*}{ Palmitic acid $(16: 0)$} & $1990-1991$ & 673.69 & $583.74,777.50$ & & 27.56 & $25.25,30.07$ & & 674.57 & $613.94,741.20$ & & 26.69 & $26 \cdot 22,27 \cdot 17$ & \\
\hline & 1999-2000 & 844.79 & $737.53,967.65$ & & 26.94 & $24.80,29.26$ & & 802.82 & $726 \cdot 39,887 \cdot 29$ & & 28.65 & $28 \cdot 11,29 \cdot 19$ & \\
\hline & $2007-2008$ & $789 \cdot 31$ & $704.69,884.08$ & 0.138 & 27.66 & $25.81,29.64$ & 0.896 & 833.65 & $752.96,922.99$ & 0.002 & 26.56 & $26.05,27.08$ & 0.936 \\
\hline \multirow[t]{3}{*}{ Stearic acid (18:0) } & 1990-1991 & $332 \cdot 77$ & $286 \cdot 90,385.97$ & & 15.55 & $14 \cdot 26,16 \cdot 95$ & & 377.39 & $340 \cdot 11,418.75$ & & $17 \cdot 00$ & $16 \cdot 65,17 \cdot 37$ & \\
\hline & $1999-2000$ & $390 \cdot 25$ & $339.09,449.13$ & & 14.14 & $13.03,15.35$ & & 407.08 & $364.50,454 \cdot 62$ & & 16.51 & $16 \cdot 14,16 \cdot 89$ & \\
\hline & $2007-2008$ & 391.95 & $348.55,440.76$ & 0.111 & 15.69 & $14.65,16 \cdot 80$ & 0.677 & 503.76 & $450.20,563.69$ & 0.0001 & 18.25 & $17.84,18 \cdot 68$ & $<0.0001$ \\
\hline \multirow[t]{3}{*}{ Total cis-MUFA† } & 1990-1991 & 562.28 & $511.25,618 \cdot 41$ & (1) & 26.33 & $25.61,27.07$ & . & 562.70 & $508.55,622.61$ & 0.0001 & 25.50 & $24.84,26 \cdot 17$ & -0.000 \\
\hline & 1999-2000 & $737 \cdot 16$ & $673 \cdot 62,806 \cdot 70$ & & $26 \cdot 38$ & $25 \cdot 70,27 \cdot 08$ & & $635 \cdot 61$ & $570 \cdot .84,707.73$ & & 25.98 & $25 \cdot 27,26 \cdot 71$ & \\
\hline & $2007-2008$ & 656.06 & $608.48,707.36$ & 0.041 & $26 \cdot 33$ & $25 \cdot 76,26.91$ & 0.992 & 657.37 & $589.28,733.34$ & 0.038 & 24.97 & $23 \cdot 30,24 \cdot 65$ & 0.301 \\
\hline \multirow[t]{2}{*}{ Palmitoleic acid (16:1n-9cis) } & $\begin{array}{l}1990-1991 \\
1999-2000\end{array}$ & $\begin{array}{l}30.84 \\
43.48\end{array}$ & $\begin{array}{l}27.47,34.64 \\
38.95,48.53\end{array}$ & & $\begin{array}{l}1.32 \\
1.42\end{array}$ & $\begin{array}{l}1.24,1.40 \\
1.34,1.51\end{array}$ & & $\begin{array}{l}30 \cdot 30 \\
36 \cdot 96\end{array}$ & $\begin{array}{l}27.05,33.95 \\
32.75,41.70\end{array}$ & & $\begin{array}{l}1.25 \\
1.38\end{array}$ & $\begin{array}{l}1.19,1.32 \\
1.31,1.46\end{array}$ & \\
\hline & $2007-2008$ & 35.49 & $32.37,38.90$ & 0.183 & 1.30 & $1.24,1.37$ & 0.523 & 34.00 & $30.07,38.45$ & 0.153 & 1.13 & $1.07,1.20$ & 0.022 \\
\hline \multirow[t]{3}{*}{ Oleic acid (18: $1 n-9 c i s)$} & 1990-1991 & 440.01 & $399.20,484.99$ & & 20.50 & $19 \cdot 88,21 \cdot 14$ & & 440.45 & $397.21,488.39$ & & 19.84 & $19 \cdot 25,20 \cdot 45$ & \\
\hline & 1999-2000 & 582.89 & $531.54,639.20$ & & 20.77 & $20 \cdot 18,21.38$ & & $503 \cdot 35$ & $451.03,561.73$ & & 20.48 & $19 . .83,21 \cdot 15$ & \\
\hline & $2007-2008$ & 519.98 & $481.43,561.61$ & 0.029 & 20.77 & $20 \cdot 27,21 \cdot 28$ & 0.536 & 522.96 & $467 \cdot 70,584.74$ & 0.025 & 19.96 & $18.35,19.59$ & 0.630 \\
\hline \multirow[t]{3}{*}{ Total cis-n-6 PUFA $\ddagger$} & $1990-1991$ & 461.79 & $416 \cdot 13,512.47$ & & 21.66 & $20.93,22.40$ & & 486.21 & $437.93,539.82$ & & 22.00 & $21 \cdot 30,22 \cdot 72$ & \\
\hline & 1999-2000 & 624.64 & $565.96,689.41$ & & $22 \cdot 27$ & $21.56,23.00$ & & 531.40 & $475.53,593.84$ & & 21.71 & $20 \cdot 97,22 \cdot 46$ & \\
\hline & $2007-2008$ & 555.74 & $511.79,603.46$ & 0.022 & $22 \cdot 35$ & $21 \cdot 76,22 \cdot 96$ & 0.174 & 650.35 & $580 \cdot 83,728.18$ & 0.0001 & 23.58 & $22 \cdot 77,24.41$ & 0.006 \\
\hline \multirow{3}{*}{$\begin{array}{l}\text { Linoleic acid }(18: 2 n-6 c i s, c i s, \\
\text { cis) }\end{array}$} & 1990-1991 & $266 \cdot 48$ & $239.17,296.90$ & & 12.60 & $12.07,13.14$ & & $275 \cdot 20$ & $247 \cdot 25,306 \cdot 31$ & & 12.56 & $12.05,13.09$ & \\
\hline & 1999-2000 & 383.21 & $345 \cdot 90,424 \cdot 54$ & & 13.75 & $13 \cdot 21,14 \cdot 31$ & & 314.06 & $280 \cdot 29,351 \cdot 90$ & & 12.93 & $12 \cdot 38,13.51$ & \\
\hline & $2007-2008$ & 318.52 & $292.41,346.96$ & 0.050 & 12.92 & $12.49,13.36$ & 0.596 & 348.41 & $310 \cdot 33,391 \cdot 16$ & 0.003 & 12.73 & $12 \cdot 17,13.31$ & 0.642 \\
\hline \multirow[t]{3}{*}{ Long-chain $n$-6 PUFA } & 1990-1991 & 188.53 & $169 \cdot 26,210 \cdot 00$ & & 8.76 & $8.37,9.16$ & & 204.74 & $183.08,228.96$ & & 9.16 & $8 \cdot 74,9.60$ & \\
\hline & $1999-2000$ & 234.26 & $211.51,259.46$ & & 8.27 & $7.92,8.63$ & & 208.84 & $185 \cdot 46,235 \cdot 18$ & & 8.45 & $8.04,8.88$ & \\
\hline & 2007-2008 & 228.45 & $209.77,248.80$ & 0.012 & 9.09 & $8.77,9.43$ & 0.098 & 290.55 & $257.48,327.87$ & $<0.0001$ & 10.44 & $9.92,10.98$ & 0.001 \\
\hline \multirow[t]{3}{*}{ Total cis-n-3 PUFA§ } & 1990-1991 & 66.43 & $58.41,75.55$ & & 3.27 & $3.04,3.50$ & & 80.63 & $70.64,92.03$ & & 3.78 & $3.49,4.08$ & \\
\hline & 1999-2000 & 69.65 & $61 \cdot 66,78.68$ & & $2 \cdot 60$ & $2 \cdot 43,2 \cdot 78$ & & 64.66 & $56 \cdot 19,74.42$ & & 2.76 & $2.54,3.00$ & \\
\hline & 2007-2008 & 57.05 & $51.53,63.17$ & 0.041 & 2.44 & $2 \cdot 31,2 \cdot 58$ & $<0.0001$ & 77.16 & $66.88,89.02$ & 0.588 & 2.92 & $2 \cdot 68,3.18$ & $<0.0001$ \\
\hline \multirow{3}{*}{$\begin{array}{l}a \text {-Linolenic acid }(18: 3 n-3 c i s, \\
\text { cis,cis) }\end{array}$} & $1990-1991$ & 2.46 & $1.32,4.59$ & & $0 \cdot 15$ & $0.10,0.24$ & & $2 \cdot 86$ & $1 \cdot 56,5 \cdot 24$ & & 0.17 & $0.11,0.26$ & \\
\hline & 1999-2000 & 1.68 & $0.93,3.04$ & & 0.09 & $0.06,0.144$ & & $2 \cdot 23$ & $1 \cdot 17,4 \cdot 25$ & & 0.12 & $0.08,0.20$ & \\
\hline & $2007-2008$ & 0.90 & $0.55,1.48$ & 0.011 & 0.06 & $0.04,0.09$ & 0.002 & 1.51 & $0.78,2.90$ & 0.149 & 0.08 & $0.05,0.13$ & 0.033 \\
\hline \multirow[t]{3}{*}{ Long-chain $n$-3 PUFA } & 1990-1991 & 57.69 & $50 \cdot 32,66 \cdot 14$ & & 2.89 & $2 \cdot 68,3 \cdot 10$ & & 71.86 & $62 \cdot 52,82 \cdot 60$ & & 3.40 & $3.13,3.69$ & \\
\hline & $1999-2000$ & 60.57 & $53.21,68.95$ & & 2.29 & $2 \cdot 14,2 \cdot 45$ & & 56.95 & $49.12,66.03$ & & 2.47 & $2 \cdot 26,2.69$ & \\
\hline & 2007-2008 & 50.54 & $45 \cdot 36,56 \cdot 31$ & 0.092 & $2 \cdot 20$ & $2.08,2 \cdot 33$ & $<0.0001$ & $70 \cdot 23$ & $60.41,81.64$ & 0.745 & 2.68 & $2 \cdot 46,2.93$ & $<0.0001$ \\
\hline \multirow[t]{3}{*}{$n-6: n-3$ PUFA } & 1990-1991 & $\begin{array}{r}6.95 \\
8.97\end{array}$ & $6.45,7.49$ & & 6.63 & $6 \cdot 18,7 \cdot 11$ & & $\begin{array}{r}6.03 \\
8.20\end{array}$ & $5.58,6.51$ & & $\begin{array}{l}5.83 \\
7.87\end{array}$ & $5.41,6.27$ & \\
\hline & 1999-2000 & 8.97 & $8.35,9.63$ & & 8.57 & $8.01,9.16$ & & 8.22 & $7.57,8.92$ & & 7.87 & $7 \cdot 27,8.51$ & \\
\hline & 2007-2008 & 9.74 & $9 \cdot 18,10 \cdot 34$ & $<0.0001$ & 9.17 & $8.67,9.70$ & $<0.0001$ & 8.43 & $7 \cdot 75,9 \cdot 16$ & $<0.0001$ & 8.07 & $7.45,8.74$ & $<0.0001$ \\
\hline \multirow[t]{3}{*}{ Total iTFAll } & 1990-1991 & 37.70 & $34.94,40.68$ & & 1.72 & $1.58,1.86$ & & 38.82 & $35.51,42.44$ & & 1.70 & $1.58,1.82$ & \\
\hline & 1999-2000 & 39.51 & $36 \cdot 77,42 \cdot 46$ & & 1.39 & $1.29,1.51$ & & 36.23 & $32.95,39.82$ & & 1.44 & $1.34,1.55$ & \\
\hline & $2007-2008$ & 38.07 & $35 \cdot 85,40 \cdot 43$ & 0.938 & 1.51 & $1.42,1.62$ & 0.054 & 38.61 & $35.07,42.51$ & 0.896 & 1.38 & $1.28,1.50$ & 0.0001 \\
\hline Palmitelaidic acid & $1990-1991$ & 29.80 & $27.63,32 \cdot 13$ & & 1.28 & $1 \cdot 15,1.41$ & & 31.34 & $28 \cdot 77,34 \cdot 13$ & & 1.30 & $1 \cdot 19,1.41$ & \\
\hline & 1999-2000 & 29.46 & $27.43,31 \cdot 65$ & & 0.96 & $0.88,1.06$ & & 28.82 & $26.32,31.56$ & & 1.08 & $0.98,1.18$ & \\
\hline & $2007-2008$ & 27.40 & $25.81,29.08$ & 0.069 & 1.01 & $0.93,1.09$ & 0.001 & 28.96 & $26 \cdot 40,31 \cdot 76$ & 0.205 & 0.97 & $0.88,1.06$ & $<0.0001$ \\
\hline Elaidic acid (18:1n-9trans) & 1990-1991 & 3.08 & $2.54,3.73$ & & 0.18 & $0 \cdot 16,0.20$ & & 2.13 & $1 \cdot 62,2 \cdot 81$ & & 0.13 & $0.11,0.16$ & \\
\hline & 1999-2000 & 3.86 & $3.22,4.63$ & & 0.17 & $0.15,0.19$ & & $2 \cdot 79$ & $2.08,3.73$ & & 0.14 & $0.12,0.18$ & \\
\hline
\end{tabular}




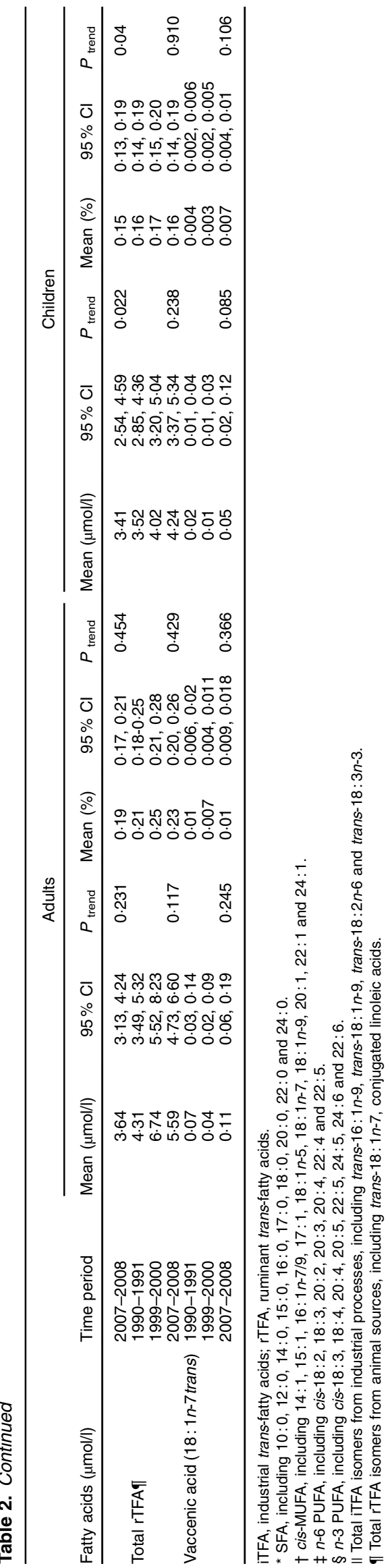

in adults and children. Levels of total iTFA - with palmitelaidic acid and elaidic acid being the major contributors to total TFA from frying oils and PHVO - remained stable over time. However, when distinguishing the two TFA isomers, levels and proportions of elaidic acid, the main TFA occurring in PHVO, increased over time in children, while levels and proportions of palmitelaidic tended to decrease over time, albeit remaining high compared with European values. This set of data might suggest that the use of PHVO has further increased in the last two decades in this rural population in Uganda.

These data indicate unfavourable trends of dietary fatty acids over time. It is tempting to hypothesise that the unfavourable trend in this population study in Uganda might reflect a global change in this population's diet and be a surrogate for an early stage of nutrition transition in general. This is in line with the emergence and popularisation of soft drinks and fast foods in sub-Saharan Africa ${ }^{(3)}$. A study of the diet of urban Ethiopian adults showed the regular consumption of oil and fat, while the consumption of fruits and vegetables decreased over time ${ }^{(19)}$. A dietary survey conducted in Kenya showed significant urban/ rural differences in the contribution of macronutrients to total energy intake, with higher energy from fat and lower energy from carbohydrates in urban areas ${ }^{(20)}$. This study might also illustrate differences in nutrition transition stages between urban and rural areas.

The specific fatty acid profile in the Ugandan population study is of concern, as some epidemiological studies reported a positive association between intake of SFA and iTFA and mortality from CHD, ischaemic stroke and type 2 diabetes ${ }^{(21)}$. Intake of iTFA, even at low levels, is specifically associated with several adverse outcomes, including inflammation and cardiovascular mortality ${ }^{(22)}$. Although limited, there is growing evidence that increasing blood proportion of iTFA is associated with an increased risk of weight gain ${ }^{(23)}$ and breast cancer ${ }^{(11,24)}$.

If iTFA are a cause of NCD in the West and yet higher in levels in Africa, where NCD are less common, this could be attributable to other characteristics of the population, which mitigate the increased risks from higher exposure to iTFA. For examples, risk factors such as smoking, physical activity and harmful alcohol intake associated with the risk of NCD have been reported to be rare in Uganda ${ }^{(25)}$. However, the impact of specific iTFA isomers on the risk of NCD has not been studied within the Ugandan population study where the proportion of some iTFA isomers is higher than that in Europe.

Key strengths of this study are the availability of blood samples collected from 1990 in adults and children. In addition, we were able to separate and quantify sixty fatty acids, including various TFA isomers from natural and industrial processes. Finally, we were able to examine the relative concentrations as well as absolute concentrations of fatty acids. Among limitations, long-term storage of blood samples might have affected fatty acids, particularly PUFA. However, samples stored between $-196^{\circ} \mathrm{C}$ and $-80^{\circ} \mathrm{C}$ are likely to be stable over time ${ }^{(26)}$.

In conclusion, these data show evidence of an unfavourable trend over time of dietary fatty acids in a rural Ugandan population characterised by increasing levels of SFA, MUFA, iTFA (specifically elaidic acid) and n-6:n-3 PUFA. If the major 
sources of these harmful fats are the types of oils and fats used for cooking, this offers opportunity for policy change to improve public health in low-income settings. Finally, we also demonstrate the power of this approach in characterising how serum fatty acid profiles have changed over time, providing a benchmark for future prospective studies and a comparator for countries at different stages of nutritional transition.

\section{Acknowledgements}

The authors acknowledge Mrs Béatrice Vozar and Siham El Manssouri at the International Agency for Research on Cancer, for their assistance with laboratory measurements of serum fatty acids.

The GPC is jointly funded by the UK Medical Research Council (MRC) and the UK Department for International Development (DFID) under the MRC/DFID Concordat agreement.

V. C. and R. N. designed the project, drafted the manuscript and contributed to the interpretation of the data. V. C. supervised laboratory analysis. L. J. G. and C. B. conducted statistical analysis. All the authors have read, corrected the drafts and approved the final version of the manuscript.

The authors declare no conflict of interest.

\section{References}

1. World Health Organization (2011) Global Status Report on Noncommunicable Diseases 2010. Geneva: WHO.

2. Popkin BM, Adair LS \& Wen NS (2012) Now and then: the global nutrition transition: the pandemic of obesity in developing countries. Nutr Rev 70, 3-21.

3. Steyn NP \& Mchiza ZJ (2014) Obesity and the nutrition transition in sub-Saharan Africa. Ann N Y Acad Sci 1311, 88-101.

4. Mattei J, Malik V, Wedick NM, et al. (2012) A symposium and workshop report from the Global Nutrition and Epidemiologic Transition Initiative: nutrition transition and the global burden of type 2 diabetes. Br J Nutr 108, 1325-1335.

5. Wiseman MJ (2015) Nutrition and cancer - global and African perspectives: a focused update. Proc Nutr Soc 74, 437-440.

6. Abrahams Z, Mchiza Z \& Steyn NP (2011) Diet and mortality rates in sub-Saharan Africa: stages in the nutrition transition. BMC Public Health 11, 801.

7. Food and Agriculture Organization (2014-2015) Food balance sheets. http://faostat3.org/faostatgateway/go/go/home/E (accessed November 2013).

8. Saadatian-Elahi M, Slimani N, Chajès V, et al. (2009) Plasma phospholipid fatty acid profiles and their association with food intakes: results from a cross-sectional study within the European Prospective Investigation into Cancer and Nutrition. Am J Clin Nutr 89, 331-346.

9. Chajès V, Biessy C, Byrnes G, et al. (2011) Ecological-level associations between highly processed food intakes and plasma phospholipid elaidic acid concentrations: results from a crosssectional study within the European Prospective Investigation into Cancer and Nutrition (EPIC). Nutr Cancer 63, 1235-1250.
10. Asiki G, Murphy G, Nakiyingi-Miiro J, et al. (2013) The general population cohort in rural south-western Uganda: a platform for communicable and non-communicable disease studies. Int J Epidemiol 42, 129-141.

11. Chajès V, Assi N, Biessy C, et al. (2017) A prospective evaluation of plasma phospholipid fatty acids and breast cancer risk in the EPIC study. Ann Oncol 28, 2836-2842.

12. Kobayashi M, Sasaki S, Kawabata T, et al. (2001) Single measurement of serum phospholipid fatty acid as a biomarker of specific fatty acid intake in middle-aged Japanese men. Eur J Clin Nutr 55, 643-650.

13. Bradbury KE, Skeaff CM, Crowe FL, et al. (2011) Serum fatty acid reference ranges: percentiles from a New Zealand National Nutrition Survey. Nutrients 3, 152-163.

14. Mancini A, Imperlini E, Nigro E, et al. (2015) Biological and nutritional properties of palm oil and palmitic acid: effects on health. Molecules 20, 17339-17361.

15. Micha R, King IB, Lemaitre RN, et al. (2010) Food sources of individual plasma phospholipid trans fatty acid isomers: the Cardiovascular Health Study. Am J Clin Nutr 91, 883-893.

16. Sansone A, Melchiorre M, Chatgilialoglu C, et al. (2013) Hexadecenoic fatty acid isomers: a chemical biology approach for human plasma biomarker development. Chem Res Toxicol 26, 1703-1709.

17. Bhardwaj S, Passi SJ, Misra A, et al. (2016) Effect of heating/ reheating of fats/oils, as used by Asian Indians, on trans fatty acid formation. Food Chem 212, 663-670.

18. Riha J, Karabarinde A, Ssenyomo G, et al. (2014) Urbanicity and lifestyle risk factors for cardiometabolic diseases in rural Uganda: a cross-sectional study. PLos Med 11, e1001683.

19. Amare B, Moges B, Moges F, et al. (2012) Nutritional status and dietary intake of urban residents in Gondar, Northwest Ethiopia. BMC Public Health 12, 752.

20. Steyn NP, Nel JH, Parker W, et al. (2011) Dietary, social, and environmental determinants of obesity in Kenyan women. Scand J Public Health 39, 88-97.

21. De Souza RJ, Mente A, Maroleanu A, et al. (2015) Intake of saturated and trans unsaturated fatty acids and risk of allcause mortality, cardiovascular disease, and type 2 diabetes: systematic review and meta-analysis of observational studies. BMJ 351, h3978.

22. Mozaffarian D, Katan MB, Ascherio A, et al. (2006) Trans fatty acids and cardiovascular disease. $N$ Engl J Med 354, 1601-1613.

23. Chajès V, Biessy C, Ferrari P, et al. (2015) Plasma elaidic acid level as biomarker of industrial trans fatty acids and risk of weight change: report from the EPIC Study. PLOS ONE 10, e0118206.

24. Hirko KA, Chai B, Spiegelman D, et al. (2018) Erythrocyte membrane fatty acids and breast cancer risk: a prospective analysis in the nurses' health study II. Int J Cancer $\mathbf{1 4 2}$, 1116-1129.

25. Nakibuuka J, Sajatovic M, Nankabirwa J, et al. (2015) Strokerisk factors differ between rural and urban communities: population survey in Central Uganda. Neuroepidemiology $\mathbf{4 4}$, $156-165$.

26. Hodson L, Skeaff CM \& Fielding BA (2008) Fatty acid composition of adipose tissue and blood in humans and its use as a biomarker of dietary intake. Prog Lipid Res 47, 348-380. 